\title{
HUMAN BABESIOSIS: A GENERAL REVIEW WITH SPECIAL REFERENCE TO EGYPT
}

\section{By}

\author{
AHMAD MEGAHED AHMAD SALEH ${ }^{1}$, SAMIA MOHAMMAD ADAM ${ }^{2}$, \\ AMR M. EL-SAYED ABDEL-MOTAGALY ${ }^{1}$, ABEER MOHAMMAD \\ ABDALLAH IBRAHIM ${ }^{1}$ and TOSSON ALY MORSY ${ }^{3}$ \\ Military Medical Academy ${ }^{1}$, Department of Nursing Administration, \\ Faculty of Nursing ${ }^{2}$, and Department of Parasitology, Faculty of Medicine ${ }^{3}$, \\ Ain Shams University, Cairo $11566^{2,3}$, Egypt
}

\begin{abstract}
Babesiosis is a tick-borne malaria-like illness caused by species of the intra-erythrocytic protozoan Babesia. Humans are opportunistic hosts for Babesia when bitten by nymph or adult ticks. Currently, Babesia infection is transmitted by various tick vectors in Europe, Asia, Africa and the northwestern and northeastern United States. Human babesiosis was first described in 1957 but is now known to have worldwide distribution. The increase in reported cases is likely due to increases in actual incidence as well as increased awareness of the disease. Despite the diagnostic and preventive advances resulting from extensive research and a greater understanding of the disease, babesiosis continues to have significant medical impact as a confounding variable in the diagnosis and treatment of Lyme disease and as a potential threat to the blood supply, especially in the United States. Diagnostic advances, like the development of PCR assays, have resulted in increased sensitivity for detection as well as the discovery and characterization of new babesial species. Further studies using the molecular tools now available and those to be developed will lead to a better understanding of the natural history of these organisms, including the transmission cycle and the potential role of Babesia parasites themselves as immunomodulator.

Human babesiosis is usually an asymptomatic infection in healthy individuals. Several patients become symptomatic, and, within these subpopulations, significant morbidity and mortality occur, especially in elderly, immunocompromised, or asplenic patients. It is difficult to diagnose. Although the index of suspicion should be high in rural Babesia endemic areas, patients with babesiosis have few, if any, localizing signs to suggest the disease. Diagnosis confirmation depends on the parasitemia degree, the expertise and well trained laboratory personnel.

Most patients infected by $B$ microti who are otherwise healthy appear to have a mild illness and typically recover without specific chemotherapy; however, treatment is recommended for all diagnosed cases to prevent sequelae and potential transmission through blood donation. In addition, patients should be advised to take precautions against tick exposure and to refrain from donating blood until completely cured of babesiosis.
\end{abstract}

Key-words: Babesiosis, Risk-factors, Tick, Blood transfusion, Needle-stick, Recommendations

\section{Introduction}

Babesiosis is a protozoan (order Piroplasmidora and family Babesiidae) tick borne illness caused by malaria-like parasites that infect red blood cells and result in hemolysis. There are more than 100 species of Babesia, all of which have an animal reservoir, typically either rodents or cattle, and are transmitted to humans via a tick vector.

Transmission: Babesiosis may also be acquired by blood transfusion or needle-stick particularly in areas endemic for B. microti (the most common worldwide) B. duncani (west coast of USA and Europe) and $B . d i$ vergens (in USA) and $B$. venatorum (Europe). The zoonotic cycle is maintained by tick vectors. Human infection is accidental; humans are not definitive reservoir hosts (Gubernot et al, 2009). Dobroszycki et al. (1999) reported an adult, a child, and four neonates infected from a single blood donation by an asymptomatic Babesia-infected donor, of which three became parasitemic.

Transfusion-associated babesiosis may be acquired throughout the year; source blood 
donors typically reside in endemic areas (Della-Giustina et al, 2005).

The rare ways of transmission are transplacental, perinatal and transfusion-associated. Sethi et al. (2009) reported two neonates with transplacentally or prenatally acquired (congenital) babesiosis and a third congenital case of babesiosis in a 26-day-old infant; transmission was determined on the basis of a blood smear from the infant $(15 \%$ parasitemia) and serologic results from the infant and mother.

Infection of the human host can cause a very severe host-mediated pathology including fever, and hemolysis leading to anemia, hyperbilirubinuria, hemoglobinuria and possible organ failure. Apparently owing to increased medical awareness and better diagnostic methods, the number of reported cases in humans is raising steadily worldwide (Hildebrandt et al, 2013).

Clinical manifestations: Babesia infections range from asymptomatic to severe and sometimes fatal. The severity of infection depends on the Babesia species and the immune status of the host. B. microti is the predominant species with infection manifestations ranged from mild to severe, sometimes resulting in death (Vannier et al, 2008).

Symptoms typically develop one to six weeks after the bite of a tick; an incubation period of 12 weeks was described in one case report. Up to two-thirds of patients do not recall a tick bite. The incubation period varies from one to nine weeks in the setting of transfusion transmitted infection (Leiby, 2006). Patients typically experience gradual onset of fatigue, malaise, and weakness. Fever $\left(>38^{\circ} \mathrm{C}\right)$ is intermittent or sustained, and may reach $41^{\circ} \mathrm{C}$. Fever is accompanied by one of more of the following: chills, sweats, headache, myalgia, arthralgia, and anorexia. Less common symptoms include neck stiffness, sore throat, dry cough, and shortness of breath, weight loss, nausea, vomiting, diarrhea, and dark urine (Hatcher et al, 2001).
On physical examination, fever is the salient feature. Mild splenomegaly and hepatomegaly may be noted. Lymphadenopathy is absent. Jaundice, slight pharyngeal erythema, retinopathy with splinter hemorrhages and retinal infarcts are rare. However, concomitant or serial infection due to TOBB may be common in tick exposed patients with various tick-borne pathogens must be always considered, especially in endemic regions in the differential diagnosis of acute febrile illness following a tick bite (Leschnik et al, 2013).

In the normosplenic patients, parasitemia ranges from 1 to $20 \%$. Mild babesiosis typically is associated with parasitemia $<4$ to $5 \%$. Low hematocrit, low hemoglobin, elevated total bilirubin, low haptoglobin, and/ or reticulocytosis are indicative of hemolytic anemia. The thrombocytopenia is common. White blood cell counts are normal, increased, or mildly decreased. Liver enzymes are elevated as alkaline phosphatase, aspartate aminotransferase, alanine aminotransferase, and lactic dehydrogenase (Krause et al, 2000).

Role of the spleen in host defense: The sinusoids of the spleen serve as a "fine tooth comb" to filter blood through a series of capillaries as small as 1 micron in diameter to sequester senescent, rigid erythrocytes from the circulation. The mononuclear phagocytes located within this capillary network also ingest circulating bacteria, particularly nonopsonized organisms, thus cleansing the roughly 6 percent of cardiac output which perfused the spleen. The spleen also serves as the largest lymphoid organ within the body and contains nearly half of the body's total immunoglobulin-producing B lymphocytes. Thus, the spleen not only clears bacteria from the circulation but also processes this foreign material to stimulate the production of opsonizing antibody. This function is particularly important in the clearance of encapsulated organisms (Chiossone et al, 2012). 
Individuals who are asplenic or have impaired splenic function are at increased risk of developing life-threatening infections as babesiosis and more especially due to encapsulated bacteria. This risk is higher in children, but adults can also develop fulminant infection or "post splenectomy sepsis" (PSS). Cryptococcus neoformans is an encapsulated yeast usually causing infection in immunocompromised patients. In a review of cryptococcal infection in HIV-negative patients, splenectomy was reported to be a risk factor for infection in 3\% of cases. Detailed case reports are lacking. a case of disseminated $C$. neoformans infection in a patient who had the splenectomy performed for warm autoantibody haemolytic anaemia some months before he presented with signs and symptoms of meningitis was reported (Qazzafi et al, 2007). Fever or rigors should be considered the first warning signs of possible post-splenectomy sepsis. The broadspectrum antibiotics must be initiated promptly since clinical deterioration can occur over hours. Antibiotic administration should not be delayed by a diagnostic work-up (Clement et al, 2015).

Most mild B. microti infections are selflimited or respond to a standard 7 to 10 day antimicrobial regimen. Parasitemia may persist in some patients, despite lack of symptoms. Illness may recrudesce upon immunosuppression induced by cancer chemotherapy or transplantation. Severe babesiosis was defined as a hospitalization ending in death, lasting longer than two weeks, or requiring a stay in the ICU of two days or longer associated with parasitemia $>4 \%$, alkaline phosphatase $>125 \mathrm{U} / \mathrm{L}$, and white blood cell counts $>5 \times 10 /$ L. Elevated alkaline phosphatase levels, elevated white blood cell counts, and male gender were strong predictors of severe outcome. Patients may be presented with malaise, arthralgia or myalgia, and shortness of breath, thrombocytopenia and elevated liver enzymes are also common. Risk factors for developing severe illness due to babesiosis include: Age
$>50$ years splenectomy co-infection with HIV or Borrelia burgdorferi immunosuppression caused by cancer chemotherapy or transplantation (Florescu et al, 2008). The complications as acute respiratory distress syndrome disseminated intravascular coagulation, congestive heart failure, renal failure, myocardial infarction, splenic infarcts or splenic rupture associated with severe anemia (hemoglobin $\leq 10 \mathrm{~g} / \mathrm{dl}$ ), and to a lesser degree with the high parasitemia $(\geq 10 \%)$, which poorly correlates with the anemia degree. Immunocompromised individuals may experience persistent or relapsing babesiosis (Florescu et al, 2014).

Besides, all symptomatic cases attributed to the $B$. divergens occurred among asplenic individuals and among normosplenic immunocompetent patients and appear with abrupt onset following an incubation period of one to three weeks. The presenting symptoms are hemoglobinuria and jaundice; these are indicative of severe intravascular hemolysis. Other symptoms include persistent high fever $\left(>41^{\circ} \mathrm{C}\right)$, shaking chills, intense sweats, headache, myalgia, and lumbar and abdominal pain. On physical examination mild hepatomegaly may be noted. The natural history of untreated infection includes renal failure and pulmonary edema. Death is the outcome in about 40 percent of cases. Abnormal laboratory findings include low hemoglobin levels (4 to $8 \mathrm{~g} / \mathrm{dL}$ ). In the absence of a functional spleen, parasitemia can be as high as 80 percent (Froberg et al, 2008).

All the $B$. divergens-like organisms patients were asplenic men aged $>50$ years and required hospitalization, at admission included fever $\left(>38^{\circ} \mathrm{C}\right)$ and thrombocytopenia, total bilirubin and lactate dehydrogenase levels were elevated. The peak parasitemia ranged from 20 to 415 . Sometimes, patients developed no complications or developed renal failure but recovered following therapy, exchange transfusion, and hemodialysis, or died despite medical care (Kuwayama and Briones, 2008), disseminated coagulation, pulmonary edema, and renal insuffi- 
ciency, but recovered upon therapy (Homer et al, 2000).

Clinical manifestations of $B$. duncani and related organisms are similar to those of $B$. microti infection, including the weakness, malaise, fatigue, fever, chills, sweats, headache, myalgia, arthralgia, nausea, vomiting, anorexia, weight loss, and dark urine. The laboratory findings might include the low hematocrit, thrombocytopenia, hyperbilirubinemia, and elevated liver enzymes. Besides, the blood donors had no significant clinical history; the other had reported slight nausea lasting 10 days around the time of blood donation (Hunfeld et al, 2008).

Diagnosis: Babesiosis diagnosis of should be considered in the following circumstances: Patients with flu-like symptoms in the setting of appropriate exposure (e.g., residents of endemic areas, or travelers returning from endemic areas) Patients presenting with Lyme disease or human granulocytic anaplasmosis and other tick-borne infectious diseases (Alcoba and Chappuis, 2015)

Microscopy: The definitive diagnosis of babesiosis must be carried out by the technical microscopic examination of thin blood smears (Wright or Giemsa staining under oil immersion). Thick blood smears are not recommended, as babesial parasites may be too small to be visualized. Identification of the parasite may require multiple blood smears over several days if the parasitized red blood cells are rare; particularly at the onset of symptoms (Park et al, 2015).

B. microti appear round, oval or pearshaped. The most common form is the ring, with a pale blue cytoplasm and one or two red chromatic dots. Multiple infections per cell may be observed. Ring forms may be mistaken for Plasmodium falciparum trophozoites. Distinguish-ing features of Babesia include: Occasional merozoites arranged in tetrads, referred to as "Maltese Cross" Occasional exoerythrocytic parasites (when parasitemia is high) Absence of brownish pigment deposits (hemozoin) in ring forms $\mathrm{Ab}$ sence of schizonts and gametocytes. B. di- vergens and $B$. divergens-like organisms appear round or pear-shaped. Paired piriforms assemble by their pointed extremities with an angle that is obtuse, an arrangement not seen with $B$. microti. In human red blood cells, paired piriforms of $\mathrm{B}$. divergens are typically located in a central or subcentral position, and do not protrude at the cell surface. $B$. divergens-like organisms may be in the accole position (e.g., at the margin of the red blood cell). In human red blood cells, $B$. divergens and $B$. divergens-like organisms may also arrange in tetrads. B. duncani trophozoites appear round to oval, or pearshaped, and are indistinguishable from those of B. microti. Tetrads of B. duncani, however, are not as rare as those of B. microti. $B$. venatorum, like $B$. divergens, appear round or pear-shaped. The tetrads might be observed (Rosenblatt, 2009).

The evaluation of smears requires time and expertise. Clinicians should inquire whether laboratories are staffed with expert personnel. Automated cell readers should not be used, as they fail to detect merozoites in red blood cells. Uncertainty as to the presence of Babesia or Plasmodium should prompt empiric management for both entities. Na et al. (2014) reported that clinicians have difficulties in making a falciparum malaria clinical diagnosis as well as in having accessibility to effective anti-malarial agents. They described an unusual case of imported falciparum malaria with severe hemolytic anemia lasting over 2 weeks, clinically mimic-king a coinfection with babesiosis. The patient was diagnosed with severe falciparum malaria in France after traveling to the Republic of Benin, West Africa. He received a 1-day course of intravenous artesunate and a 7-day course of Malarone (atovaquone/proguanil) with supportive hemodialysis. Coming back to Korea 5 days after discharge, he was readmitted due to recurrent fever, and further treated with Malarone for 3 days. Both the peripheral blood smears and PCR test were positive for Plasmodium falciparum. However, he had prolonged severe hemolytic 
anemia ( $\mathrm{Hb} 5.6 \mathrm{~g} / \mathrm{dl})$. Therefore, 10 days after the hospitalization, Babesia was considered to be potentially coinfected. A 7-day course of Malarone and azithromycin was empirically started. The patient became afebrile within 3 days of this babesiosis treatment, and hemolytic anemia profiles began to improve at the completion of the treatment. He has remained stable since his discharge. PCR assays failed to detect DNA of Babesia spp. from blood. In addition, during the retrospective review of the case, the artesunate-induced delayed hemolytic anemia was considered as an alternative cause of the unexplained hemolytic anemia.

1- PCR-based amplification of the babesial $18 \mathrm{~S}$ rRNA gene is more sensitive than blood smear examination and results can be available within 24 hours (Persing et al, 1992). The amplification of full-length 18S rRNA gene is required for phylogenetic characterization of the Babesia species (Birkenheuer et al, 2006)

PCR is especially useful in low parasitemia setting. Wilson et al. (2015) developed a droplet digital for detection of Babesia is microscopic examination of blood smears. Recent PCR-based assays, including realtime PCR, have been developed for B. microti. On the other hand, molecular assays that detect and distinguish between B. microti and $B$. duncani infections are lacking. Closely related species of Babesia can be differentiated due to sequence variation within the internal transcribed spacer (ITS) regions of nuclear ribosomal RNAs. They concluded that methods could be used as sensitive approaches to monitor the progression of parasitemia in rodent models of infection as well as serve as suitable molecular tests in blood screening

2- Serologic diagnosis with the indirect immunofluorescent antibody testing (IFAT) is most useful when parasites are not visualized by microscopy and DNA is not detected by PCR. Such cases include the asymptomatic blood donors implicated in transfusion-acquired babesiosis, and asymptomatic individuals who were infected but cleared the parasites with or without treatment.

Vercammen et al. (1995) experimentally inoculated Five Naive Beagle dogs with two stocks of Babesia canis. Dogs were examined regularly for parasitaemia and for antibodies, using the indirect immunofluorescent antibody test (IFAT). This test proved to be useful for the diagnosis of babesiosis. After primo-infection dogs seem to develop a certain degree of immunity, although this immunity is neither absolute nor of long duration. Treatment of infected dogs with imidocarb $(6 \mathrm{mg} / \mathrm{kg})$ cleared the infection, but did not prevent the production of IFATantibodies. An infected dog treated with long acting oxytetracycline $(20 \mathrm{mg} / \mathrm{kg})$ became a subclinical, chronic carrier of the disease. Nayel et al. (2012) evaluated the direct microscopy (blood smears), indirect fluorescent antibody test (IFAT) and polymerase chain reaction (PCR). Giemsastained blood smears revealed that, out of 405 examined cattle, 33 (8.15\%) were infected with Babesia sp. and 65 (16.05\%) with Theileria sp. (total number of infected cattle was 98). Statistically, there was a nonsignificant difference of the positivity in response to the three techniques; thus, any of these methods can be described as useful for diagnosing blood parasites in both domesticated animals and birds. On the basis of the obtained results, it could be concluded that direct microscopy can be used in acute infections, whereas IFAT and PCR are useful in chronicity.

Since an indirect fluorescence immunoassay (IFA) for the detection of specific antibodies against Babesia divergens in human sera is not commercially available, an inhouse prepared $B$. divergens IFA for the examination of bovine sera was established for serological studies in humans. Duh et al. (2007) reported that the evaluation and standardization of a $B$. divergens IFA for testing human sera is critical and that different $B$. divergens antigens provide different end-point titres of antibodies, leading to 
false negative or positive results. Serological cross-reactivity between $B$. divergens and Babesia EU1 must be taken into account when interpret-ting IFA results.

Treatment of $B$. microti mild illness: Treatment with antimicrobial therapy is appropriate in the following circumstances: Symptomatic patients with babesial parasites on blood smear or babesial DNA detected by PCR. The asymptomatic individuals with babesial parasites on the blood smear or babesial DNA detected by PCR for $\geq 3$ months Individuals with babesial parasites on blood smear or babesial DNA detected by PCR for $\geq 3$ months after initiation of therapy. Treatment with antimicrobial therapy is not appropriate in the following circumstances: Symptomatic individuals with no babesial parasites on blood smear and no babesial DNA detected by PCR, even in the presence of Babesia specific antibodies. The asymptomatic individuals within the first three months following detection of babesial parasites on blood smear or babesial DNA by PCR (Weiss et al, 2001).

The two major antimicrobial regimens consisted of atovaquone plus azithromycin or quinine plus clindamycin. Wormser et al. (2010) stated that babesiosis is an emerging tickborne malaria-like infection principally caused by Babesia microti. This infection typically resolves either spontaneously or after administration of a 7-10-day course of azithromycin plus atovaquone or clindamycin plus quinine. Although certain the highly immunocompromised patients may respond sub-optimally to these drug regimens, unlike the situation with malaria there has been no reported evidence that the cause of treatment failure is infection with drug-resistant strains of $B$. microti. They concluded that the $B$. microti may become resistant to azithromycin-atovaquone during the treatment of babesiosis with this combined drug regimen in highly immunocompromised patients. Although research is still needed to determine the optimal therapy for highly immunocompromised patients with babesiosis, reducing the level of immunosuppression when possible would appear to be a desirable strategy.

Krause et al. (2000) reported adverse effects by $15 \%$ of the subjects who received atovaquone and azithromycin, as compared with $72 \%$ of those who received clindamycin and quinine $(\mathrm{P}<0.001)$. The most common adverse effects with atovaquone and azithromycin were diarrhea and rash (each in $8 \%$ of the subjects); with clindamycin and quinine the most common adverse effects were tinnitus $(39 \%)$, diarrhea $(33 \%)$, and decreased hearing (28\%).

Response to therapy: Symptoms should begin to improve within 48 hours of antimicrobial therapy. Fatigue and malaise may persist but should resolve fully within three months after initiation of therapy. Symptoms that persist for $\geq 3$ months should prompt repeat blood smear and/or PCR. If parasites were detected by microscopy or Babesia DNA was detected by PCR, a second course of antimicrobial therapy should be administered. The second course may consist of the same regimen but should be administered for $\geq 6$ weeks, including two weeks after parasites are no longer detected (Vial and Gorenflot, 2006).

Co-infection with other tick-borne illnesses: Patients with babesiosis in regions endemic for Lyme disease or human granulocytic anaplasmosis should be evaluated for co-infection with Borrelia burgdorferi or Anaplasma phagocytophilum, respectively. Cats are infected by Anaplasma phagocytophilum and Borrelia burgdorferi when exposed to the infected Ixodes scapularis (black-legged ticks) and Lappin et al. (2015) suggested that a PCR assay should be considered in acutely ill cats with suspected anaplasmosis that are seronegative.

Rashes in patients experiencing babesiosis are suggestive of undercurrent Lyme disease. Bratton and Corey (2005) in USA reported that tick-borne diseases in the United States include Rocky Mountain spotted fever, Lyme disease, ehrlichiosis, tularemia, babesiosis, Colorado tick fever, and relaps- 
ing fever. It is important for family physicians to consider these illnesses when patients present with influenza-like symptoms. A petechial rash initially affecting the palms and soles of the feet is associated with the Rocky Mountain spotted fever, whereas erythema migrans (annular macule with central clearing) is associated with Lyme disease. Various other rashes or skin lesions accompanied by fever and influenza-like illness also may signal the presence of a tick-borne disease. They concluded that if no symptoms follow exposure to tick bites, empiric treatment is not indicated. The same tick may harbor different infectious pathogens and transmit several with one bite. Advising patients about prevention of tick bites, especially in the summer months, may help prevent exposure to dangerous vector-borne diseases. Vannier and Krause (2009) stated that the people over the age of 50 years and immunocompromised individuals are at the highest risk of severe disease, including those with malignancy, HIV, lacking the spleen, or receiving immunosuppressive drugs. The asymptomatic carriers present a blood safety risk when they donate blood. They concluded that specific antimicrobial therapy consists of atovaquone and azithromycin or clindamycin and quinine. Exchange transfusion is used in severe cases. The use of multiple prevention strategies is recommended and consists of personal, residential, and community approaches.

Chabria and Ogbuagu (2015) reported that multiple tick-borne illnesses in a vulnerable host, in this case an elderly patient with liver cirrhosis, as risk factors for severe morbidity and potentially fatal outcomes. Thus, the patients with severe babesiosis require hospitalization. Potential complications include acute respiratory distress syndrome, disseminated intravascular coagulation, congestive heart failure and renal failure. Treatment of severe babesiosis must be tailored to patient circumstances, because the underlying comorbiddity and complication(s) of babesiosis may vary (Panduranga and Kumar, 2014)
Antimicrobial therapy: Shih and Wang (1998) reported the ability of azithromycin in com-bination with quinine to eliminate the Babesia infection in a native Taiwanese woman. The failure of elimination of the babesial infection was observed two weeks after treating with standard regimen of oral quinine plus intravenous clindamycin for a 10-day course of therapy. Azithromycin in place of clindamycin was administered for another 10-day course of therapy two months following initial treatment. Clearance of Babesia parasites was observed and verified by hamster inoculation. Vial and Gorenflot (2006) reported that there were a number of effective babesiacides, but imidocarb dipropionate (which consistently clears the parasitae-mia; often the only available drug on the market) and diminazene aceturate are the most widely used. Some Babesia spp. can infect humans, particularly Babesia microti and B. divergens, and human babesiosis is a significant emerging tick-borne zoonotic disease. The clinical manifestations differ markedly between European and North American diseases. In clinical cases, a combination of clindamycin and quinine is administered as the standard treatment, but also administration of atovaquone-azithromycin is successful. Supportive therapy such as intravenous fluids and blood transfusions are employed when necessary. More specific fast-acting new treatments for babesiosis have now to be developed. This should be facilitated by the knowledge of the Babesia spp. genome and increased interest for this malaria-like parasite.

Vyas et al. (2007) reported a patient with acquired immune deficiency syndrome presented with babesiosis six months after presumed tick exposure. Despite initial treatment with azithromycin and atovaquone, followed by quinine and clindamycin, he experienced an increasing parasite load. Finally, red blood cell exchange transfusion, anti-Babesia therapy, and the addition of the atovaquone-proguanil to the treatment regi- 
men led to symptomatic improvement and elimination of parasitemia. Low-level parasitemia recurred 20 weeks later and was eradicated by the administration of atovaquone-proguanil monotherapy. Atovaquoneproguanil appears to have activity against babesiosis and should be studied as a potential therapy for patients with the refractory babesiosis.

El Bahnasawy and Morsy (2008) presented an Egyptian human babesiosis. The signs and symptoms, $\mathrm{CBC}$, liver functions and kidney functions tests and all other serologic tests did not give any definite diagnosis. Also, he was sero-negative for malaria infection. The patient was critically diagnosed by the demonstration of the typical ring forms of Babesia species in stained blood smears. $\mathrm{He}$ was successfully treated with quinine and clindamycin, and was discharged from the hospital after the clinical and parasitological improvement. Persistent and relapsing babesiosis typically occurs in patients who are immunocompromised by the following: malignancy, asplenia, immunosuppressive therapy or HIV/AIDS. In a retrospective case control study comparing 14 patients with persistent parasitemia and babesial illness (despite repeated courses of antimicrobial therapy) to 46 control patients (who cleared parasites and resolved symptoms within one month following a single course of standard antimicrobial therapy), the case patients required treatment for $\geq 6$ weeks to achieve cure, including two weeks after parasites are no longer detected on blood smear (White, 2007).

Alternative regimens: For circumstances when clindamycin plus quinine is not effective in clearing $B$. microti (or is poorly tolerated), alternative regimens may be considered (based on case report experience): Two patients who were refractory to clindamycin plus quinine were treated successfully with azithromycin plus quinine (Shaio and Yang, 1997). The recurrence of symptoms in an AIDS patient was successfully treated with high doses of clindamycin plus azithromycin added to doxycycline after an allergy to the quinine developed. Falagas and, Klempner (1996) reported a case of babesiosis in a patient infected with HIV who presented with a prolonged fever of unknown origin; the patient had not undergone splenectomy. The parasitemia persisted despite initial clinical improvement after treatment with quinine and clindamycin. Babesiosis was controlled with a maintenance regimen consisting of clindamy-cin, doxycycline, and high-dose azithromycin, but the infection was not eradicated. They added that babesiosis should be considered in the differential diagnosis of HIV-infected patients with fevers and/or anemia in areas where the infection is endemic. HIV-infected patients who are severely immunosuppressed, even those without a history of splenectomy, may present with severe manifestations of babesiosis and develop a chronic infection, which may require therapy to prevent relapse of disease. Other antimalarial and antiprotozoal drugs proved largely unsuccessful. These included the following drugs: chloroquine, primaquine, quinacrine, pyrimethamine, pyrimethamine-sulfadoxine, artesunate, sulfadiazine, tetracycline, minocycline, pentamidine and trimethoprim-sulfamethoxazole (Zintl et al, 2003).

Persistent and relapsing babesiosis: Persistent and relapsing babesiosis typically occurs in patients who are immunocompromised by the following conditions: malignancy, asplenia, immunosuppressive therapy or HIV/AIDS (Krause et al, 2008). In a retrospective case control study comparing 14 patients with persistent parasitemia and babesial illness (despite repeated courses of antimicrobial therapy) to 46 control patients (who cleared parasites and resolved symptoms within one month following a single course of standard antimicrobial therapy), the case patients required treatment for $\geq 6$ weeks to achieve cure, including two weeks after parasites are no longer detected on blood smear (Stowell et al, 2007). 
Several drug regimens have been used for treatment of persistent and relapsing babesiosis. In addition to standard combinations (atovaquone plus azithromycin and quinine plus clindamycin), these regimens are atovaquone plus azithromycin plus doxycycline; atovaquone plus clindamycin plus doxycycline; atovaquone plus artemisinin plus doxycycline, with or without clindamycin; atovaquone plus clindamycin, with or without azithromycin.

Exchange transfusion: Partial or complete red cell exchange transfusion is appropriate for patients with high-grade parasitemia $(\geq$ $10 \%$ ), severe anemia (hemoglobin $\leq 10 \mathrm{~g} /$ $\mathrm{dL}$ ), or pulmonary, renal or hepatic compromise. Exchange transfusion is intended to clear the systemic compartment of infected red blood cells, to remove inflammatory mediators and toxic by-products of cell lysis, and to correct anemia. Management of exchange transfusion should be handled in close consultation with experts in hematology and pheresis. A single red cell exchange may be adequate, but multiple exchanges may be needed. The hematocrit and percent parasitemia should be monitored daily or every other day until parasitemia is $<5 \%$ (Wormser et al, 2006).

Treatment of $B$. Divergens Infection: $B$. divergens infection should be suspected when per-sons with exposure to endemic areas of Europe (residents or travelers returning from endemic areas) present with a sudden onset of hemoglobinuria and jaundice, particularly in the setting of asplenia. Because $B$. divergens frequently causes fulminant illness, babesiosis attributed to $B$. divergens is considered a medical emergency and should be treated with a combination of exchange transfusion and antimicrobial therapy.

Oral quinine or intravenous quinidine combined with intravenous clindamycin has been the longstanding regimen of choice. Cases of successful exchange transfusion and clindamycin monotherapy have also been reported. Other antimicrobial regimens have been successful for treatment of $B$. $d i$ vergens infection without concomitant exchange transfusion: A case of mild infection was successfully treated with intravenous pentamidine plus oral trimethoprim-sulfamethoxazole. Two patients were successfully treated with imidocarb diproprionate, the most effective treatment for $B$. divergens infection in cattle (Herwaldt et al, 2004). However, the imidocarb is not licensed for use in humans, and its use required a special license. Following clearance of parasites and resolution of symptoms, anemia may persist for $>1$ month; this should be managed by blood transfusion (Aufranc et al, 2002).

Treatment of Other Babesia Species Infection:

$B$. divergens-like organisms: Clinical experience with treatment of infection due to $B$. divergens-like organisms (in the Midwest and on the west coast of the United States) is limited to case reports. Oral quinine or quinidine (650 $\mathrm{mg}$ thrice daily) plus intravenous clindamycin (650 to $900 \mathrm{mg}$ thrice daily or $1200 \mathrm{mg}$ twice daily) was used. Hemolytic anemia was managed with transfusion; hemodialysis was instituted for acute renal failure. Following hospitalization, the antimicrobial regimen was switched to oral quinine plus oral clindamycin (Beattie et al, 2002).

$B$. duncani: The clinical experience with treatment of infection due to $B$. duncani is limited to case reports on the west coast of the United States. All reported cases of B. duncani infection have been treated with a course of quinine or quinidine (600 to 650 $\mathrm{mg}$ thrice daily) plus clindamycin $(600 \mathrm{mg}$ three to four times per day or $1200 \mathrm{mg}$ twice daily). In one case, symptoms relapsed and were successfully treated with intravenous clindamycin (1200 mg twice daily). In severe cases, the red blood cell transfusion and hemodialysis were instituted (Persing et al, 1995).

B. venatorum: Hildebrandt and Hunfeld (2014) in Germany stated that the human babesiosis is a zoonotic disease of world- 
wide increasing importance according to the increasing number of the immunocompromised patients. Clinical symptoms have a wide range from asymptomatic to severe and letal cases. So far, the detection of the parasites in the ticks and sero-epidemiological data in Europe identified 3 humanpathogenic species: $B$. microti, $B$. di-vergens und $B$. venatorum (EU1-3). The relative small number of approximately 50 documented human cases is probably due to lack of knowledge of the disease and the availability of diagnostic tools. Comprehensive systematic investigations of the prevalence in ticks, seroepidemiological data and improved diagnostic tests are urgently needed to evaluate the importance of the parasite.

Prevention: Personal protective measures that minimize the exposure to Ixodid ticks are currently the best form of prevention (Vazquez et al, 2008).

1- Protective clothing: People who venture into tick habitat are encouraged to wear light-colored clothing that covers their skin as much as possible, such as long pants and long-sleeved shirts (Plate 66, Stafford). Light-colored clothing allows easier visualization of questing ticks, thus facilitating tick removal before attachment, and the covering of skin surfaces provides a mechanical barrier to tick attachment (Hayes and Piesman, 2003).

2- Tick repellents: The effectiveness of tick repellents is probably most limited by lack of thoroughness of application. Over $70 \%$ in three Connecticut communities' residents reported using repellents when outdoors, although less than $30 \%$ reported always practicing this strategy. A case-control study found no effectiveness of repellent use in preventing Tick-borne diseases, but the numbers of both cases and controls who used repellent were low. A subsequent larger study indicated that use of repellent had an effectiveness of $20 \%$ in preventing disease. The toxicities of DEET and other repellents are low when applied according to the manufacturer's directions and published guideli- nes (Katz et al, 2008)

3- Environmental Interventions: The tick vectors of diseases spend part of their life cycle questing on vegetation to find hosts, a brief period of time feeding on hosts to obtain a blood meal, and a large part of their life span resting in leaf litter or soil. Environmental interventions aimed at reducing the number of infected immature stages and mature ticks, which are the principal vectors of diseases, can be separated into three main approaches: methods that target the tick directly, methods that target the tick or infective stages through the reservoir hosts, and landscape management to reduce human exposure to ticks (Eisen et al, 2013).

4- Targeting the tick: Traditional methods for controlling ticks include pesticides dispersion that kills ticks (= acaricides) onto vegetation over a wide area. This approach to killing ticks proved to work quite well against the tick-borne-diseases. The chemical acaricides such as carbaryl, cyfluthrin, or deltamethrin reduced the number of questing nymphal tick by 68 to $100 \%$ when applied to properties in endemic regions. In addition, a single well-timed application can be sufficient to reduce the population of questing ticks for most of the disease transmission season which differs from one country to another even in the same country (Dietrich et al, 2006).

However, many residents of regions hyperendemic for tick-borne disease in the eastern United States were reluctant to use chemical acaricides due to fears of environmental contamination and toxicity to family members and pets. Much research provided alternatives to chemical acaricides for killing ticks on vegetation. Chemicals derived from botanical sources, so called "natural products," hold promise as both tick repellents and acaricides, and certain fungi (Metarhizium anisopliae) have also been demonstrated to kill ticks (Golo et al, 2015).

5- A large variety of other biological agents, including the parasitic wasps, nematodes, and preda-tors (usually ants or beetles) have 
been shown to kill ticks (Abdelrahman et al, 2015). The main challenge for practical application of alternative tick control methods is to achieve commercial development of products that will be inexpensive, widely available, and accepted by both regulatory bodies and individuals (Panella et al, 2008).

6- Targeting reservoir hosts: There are three stages of the tick life cycle: the larva, nymph, and adult. In general, the immature stages (larva and nymph) feed on a variety of hosts including rodents, insectivores, birds, medium-sized mammals (e.g., raccoons, skunks, and opossums), and deer. In contrast, the adults feed only on larger hosts, principally the white-tailed deer. These interactions make host-targeted approaches tempting as Lyme disease prevention strategies (Stafford, 2009).

7- Trans-generational immune: Curno et al. (2011) stated that natural selection favor parents to adjust their offspring's life-history strategy and resource allocation in response to changing environmental and social conditions. Pathogens impose particularly strong and variable selective pressure on host life histories, and parental genes will benefit if offspring are appropriately primed to meet the immunological challenges ahead. They studied trans-genera-tional immune priming by examining reproductive resource allocation by female mice in response to $B$. microti prior infection to pregnancy, which mothers spent more time nursing their offspring. These offspring generated an accelerated response to $B$. microti as adults, clearing the infection sooner and losing less weight as a result of infection and showed an altered hormonal response to novel social environments, decreasing instead of increasing testosterone production upon social housing. They concluded that strategic maternal invest-ment in response to an infection led to increased disease resistance in following generation. They added that benefits of accelerated clearance of infection and reduced weight loss during infection may outweigh costs associated with reduced social domi- nance in an environment where the risk of disease is high.

\section{Nursing Role}

Fell (2000) stated that although Lyme disease is not fatal, it can cause musculoskeletal, neurological, and cardiovascular manifestations that may be difficult to treat. She added that the clinicians and nursing staff must also be aware of other potentially fatal tick-borne diseases such as babesiosis, ehrlichiosis, and Rocky Mountain spotted fever. Early identification and treatment of tickborne diseases are crucial to preventing devastating sequelae.

Undoubtedly, vector repellent is one element in the prevention of vector-borne diseases such as West Nile virus, eastern equine encephalitis, Lyme disease, malaria, dengue hemorrhagic fever, yellow fever, babesiosis, Crimean-Congo hemorrhagic fever, Rocky Mountain spotted fever, Southern tick-associated rash illness, ehrlichiosis, tick-borne relapsing fever, tularemia, and other insect and arthropod related diseases. Nurses and physicians can participate in a positive role by assisting families to determine the proper prophylaxis by recommending insect repellent choices that are economical, safe, and easy to use for them and their children. A holistic alternative might include the suggestion of clove oil in cases where families might have trepidations regarding using DEET for children (Shapiro, 2012).

\section{Some Selected Egyptian Publications}

Nagaty (1947) gave a list of the Egyptian species of Babesia and Theileria so far recorded. Maronpot and Guindy (1970) in Egypt identified B. gibsoni in the wild carnivores and domesticated dogs. Hafez et al. (1982) experimentally proved that Rhipicephalus turanicus transmitted Babesia ovis of sheep. Michael and El Refaii (1982) studied the efficacy of imidocarb dipropionate on Babesia ovis infection in sheep, and Michael et al. (1987) in Al-Minia Governorate reported the first case of human babesiosis.

El Bahrawy et al. (1993) reported that Babesia species transmitted by the ixodid 
ticks, which is a parasite of various mammalian hosts as bovine (B. bovis), equine ( $B$. equi), rodent (B. microti), canine (B. canis) and others. They examined a total of 398 Rattus rattus and $90 R$. norvegicus trapped in Suez, Ismailia, Port Said, North Sinai and South Sinai Governorates. The Giemsa stained blood films were taken from the tail and examined.

Morsy and Haridy (2000) reported that ivermectin (Ivomec) proved to be effective in controlling the dog tick $R$. Sanguineus, the vector of babesiosis.

El Kammah et al. (2001) on different Egyptian farm animals and domestic birds reported Hyalomma dromedarii, H. impeltatum, $H$. anatolicum excavatum, $H$. a. anatolicum, H. truncatum, H. m. marginatum, $H$. m. rufipes, H. m. turanicum, Boophilus annulatus, $R$. sanguineus, $R$. turanicus, $R$. guilhoni, R. camicasi, Amblyomma lipidum, A. marmoreum, A. vareigatum, Argas persicus, A. hermanii and A. arboreus in Giza, Sharkia, Ismailia, El Beheira and Sinai Governorates. Examination of camels, cows, sheep, and chickens infested with ticks showed Theileria annulata (rod and ovoid), B. bigemmina (ring), B. ovis (ovoid), and Babesiosoma gallinarum (dot-like), respectively.

Marathe et al. (2005) stated that most cases of human Babesial infections were acquired in temperate regions of the United States, Europe, France and England but few cases were described in other parts of the world, including China, Taiwan, Egypt, South Africa, and Mexico and first case of human babesiosis, in a normosplenic, previously healthy individual from India.

Mazyad and Khalaf (2002) in North Sinai Governorate reported B. ovis and Theileria ovis in sheep, goats, cattle and camels. Double infection was detected. The ticks were $R$. appendiculatus, $R$. bursa, $R$. turanicus and Haemaphysalis parva on sheep, Hyalomma anatolicum excavatum and Haemaphysalis sulcata on goats, Hyalomma lusitanicum on cattle and Hyalomma dromedarii, H. impeltatum, $H$. marginitum and $H$. a. anatolicum on camels. The B. ovis and/or Th. ovis were found in ticks gut and/or salivary glands in $R$. appendiculatus $(20.0 \%), \quad R$. bursa (16.7\%), R. turanicus (10\%), Haemaphysalis parva $(10 \%), H$. a. excavaium (30\%), $H$. dromedarii (18\%), and H. a. anatolicum (6.7\%).

Farah et al. (2003) serologically reported natural B. equi in Egyptian horses.

El Kammah et al. (2007) in Egypt reported Baberia bigemina, B. canis; Theileria annulata, and rickettesia, Aegyptianella pullorum in the saliva and the mid-guts smears of eight ixodid and two argasid tick species. They added that bacterial disease agents: Staphylococcus, Streptococcus and Yersinia were detected in salivary glands and mid-gut of eight ixodids and one argasid tick species; Bacillus was only found in Hyalomma. anatolicum excavatum, (Koch). Escherichia coli were isolated only from Argas. persicus (Oken) midguts.

El Bahnasawy and Morsy (2008) presented the second Egyptian human babesiosis. The signs and symptoms, CBC, liver functions and kidney functions tests and all other serologic tests did not give any definite diagnosis. Also, the patient was sero-negative for malaria infection. The patient was critically diagnosed by the demonstration of the typical ring forms of Babesia species in stained blood smears. He was successfully treated with Quinine and Clindamycin, and was discharged from the hospital after the clinical and parasitological improvement.

Saleh (2009) reported anaemia in cattle (Friesian x Egyptian native breed) infected with $B$. bigemina .

Gray et al. (2010) mentioned that babesiosis mostly occurs in the USA, but cases were reported in several European countries, in Egypt, India, Japan, Korea, Taiwan, and South Africa. The main pathological event is lysis of erythrocytes resulting in haemolytic anaemia, which in severe cases may lead to organ failure and death, particularly in the immuno-compromised patients. They added that prevention should be based primarily on 
increasing the awareness of physicians and the public to the risks, but infection from blood transfusions is particularly difficult to prevent. Expanding deer populations, resulting in wider distribution and greater abundance of ticks, heightened medical awareness, and growing numbers of immunocompromised patients are likely to result in a continuing rise of reported cases.

Mazyad et al. (2010) in Sinai found that ticks contained babesial DNA with sequence determination and analysis of amplified portions of nss-rDNA revealed their identity with $B$. bovis and a high degree of homology with $B$. bigemina and $B$. divergens. They represented the first genetic evidence of different species of Babesia and identified the role of Ixodes ricinus as a vector of zoonotic B. microti infection. Rodent isolate (HK) and American isolate (GI) were studied in transmission experiments. They used in vitro culture of zoonotic Babesia sp. EU1 from blood samples of rodent in Sinai and provided an evidence of transovarian and transstadial transmissions of the Babesia within $I$. ricinus, which emphasizes that this tick could be a vector and reservoir of EU1

Adham et al. (2010) reported that the prevalence of B. burgdorferi sensu lato (s.1.) the etiologic agent of Lyme disease among animal farms throughout Giza Governorate was $28 \%$ of examined soft and hard ticks. High infection rate $(66 \%)$ of $B$. burgdorferi s.l. was observed in both nymph and adult soft ticks Ornithodoros savignyi. Besides, the role of hard ticks as potential vectors of Lyme disease in Egypt, where the infection rate was between $0.0-50 \%$. Sequence analysis of PCR product of Borrelia burgdorferi sensu lato shares high degree of similarity in sequence compared to similar species in Gen-Bank. As the present authors mentioned in this text clinically Lyme disease and other tick-borne infectious diseases are confused

El-Bahnasawy et al. (2011) reported a fever hospital referred twelve years old boy with intermittent fever of unknown origin, whom clinical, parasitologic and serologic proved to be babesiosis. The boy acquired the infection from his pet dog which was heavily infested with Rhipicephalus sanguineus and suffered a mild feature of animal babesiosis. The patient was successfully treated with Atovaquone plus Azithromycin without relapse for one month follow up. The pet dog was sent to Governmental Veterinary Hospital at Abbassia for treat-ment from babesiosis and tick infestation. They concluded that children are at highest risk for infection because they are more likely to have close contact with pets and that the clinical manifestations are similar to malignant malaria, even in stained peripheral blood smears, which may pave the way to misdiagnosis and thus its underestimation not only in Egypt but also in other regional countries.

Mahmmod (2013) on Zagazig reported natural $B$. bovis infection in water buffaloes

Guneidy et al. (2014) in Egypt evaluated the in vitro effects of tannic acid, hematin (GST inhibitors) and different plant extracts (rich in tannic acid) on the activity of the recombinant glutathione S-transferase enzyme of the Egyptian cattle tick $R$. annulatus (rRaGST), in order to confirm their ability to inhibit the parasitic essential detoxification enzyme glutathione S-transferase. Extraction with $70 \%$ ethanol of Hibiscus cannabinus (kenaf flowers), Punica granatum (red and white pomegranate peel), Musa acuminata (banana peel) (Musaceae), Medicago sativa (alfalfa seeds), Tamarindus indicus (seed) and Cuminum cyminum (cumin seed) were used to assess: (i) inhibitory capacities of rRaGST and (ii) their phenolic and flavonoid contents. They found that the enzyme had also a peroxidatic activity (the specific activity equals $26.5 \mu \mathrm{mol} / \mathrm{min} / \mathrm{mg}$ protein). Both tannic acid and hematin inhibited rRaGST activity non-competitively with respect to GSH and competitively with respect to CDNB. While red pomegranate extracts inhibited rRaGST activity competitively with respect to GSH, uncompetitive 
inhibition was observed with respect to CDNB.

Youssef and Uga (2014) reported that some parasitic zoonoses are confined in Egyptian geographic areas, such as cutaneous leishmaniasis and zoonotic babesiosis in the $\mathrm{Si}$ nai. They concluded that prevention and control programs against sources and reservoirs of zoonoses should be planned by public health and veterinary officers based on reliable information from systematic surveillance.

Guswanto et al. (2014) evaluated a fluorescence-based method using SYBR green I stain (SG I) to screen anti-babesial agents in in vitro cultures of Egyptian B. bovis. The linearity between relative fluorescence units (RFU) and parasitemia was found to be well correlated with a 0.9944 goodness-of-fit $(\mathrm{r}(2))$ value. Subsequently, 50\% inhibitory concentration (IC50) values were calculated for 3 antiprotozoan agents, diminazene aceturate, nimbolide, \& gedunin, by this method. For diminazene aceturate and nimbolide, the IC(50)s determined by the fluorescencebased method (408 $\mathrm{nM} \& 8.13 \mu \mathrm{M}$, respectively) and microscopy (400.3 $\mathrm{nM} \& 9.4$ $\mu \mathrm{M}$, respectively) were in agreement. They suggested that the fluorescence-based method might be useful for anti-babesial drug screening and potential developed into a high-through-put screening (HTS) assay.

Abou-Laila et al. (2014) recommended the miltefosine as drug therapy in babesiosis.

Bajer et al. (2014) reported B. behnkei n. sp. is a novel species of the Duncani group isolated Dipodillus dasyurus in Sinai.

El-Bahy et al. (2015) studied the in vitro and in vivo the efficacy of three chemicals on the tick Boophilus annulatus. They found that the efficacy of deltamethrin was increased gradually until complete cessation of ticks showed within $3^{\text {rd }}$ day post-treatment (d.p.t.), 100\% efficacy. But the tick population begins to reappear gradually within se d.p.t.; while diazinon showed $100 \%$ efficacy at 7 d.p.t. and the ticks reappear again within14 D.P.T. The most preferred results were obtained with ivermectin which showed $100 \%$ efficacy at 7 d.p.t. and the cattle was still free from infection to 21 d.p.t. only and within 28 d.p.t.; ticks begin to reappear in slight few numbers. Thus the powerful and safe chemical which is commercially used was ivermectin.

Elsify et al. (2015) reported multiple species of Babesia and Theileria in different livestock animals in Egypt. Mahmoud et al. (2015) reported babesiosis among apparently healthy Egyptian bovines.

\section{Recommendations}

Physicians should consider babesiosis in the differential diagnosis of a febrile hemolytic disorder after blood-transfusion. The prompt diagnosis is important since babesiosis is responsive to antibiotic therapy and, untreated, can be a fatal disease in certain risk groups

Babesiosis is an infectious disease caused by protozoa of the genus Babesia. Babesia species infect vertebrate animals and cause lysis of host red blood cells. The zoonotic cycle is maintained by tick vectors. Human infection is accidental; humans are not definitive reservoir hosts. Clinical manifestations of babesiosis range from asymptomatic to severe infection (sometimes fatal). Symptoms of mild illness typically include fever, chills, sweats, headache, myalgia, arthralgia, and anorexia. Severe illness may include manifestations of brisk hemolysis as jaundice, hemoglobinuria as well as multiorgan system failure (acute respiratory distress syndrome, disseminated intravascular coagulation, congestive failure, and renal failure).

The severity of infection depends on the Babesia species and the immune status of the host. B. microti is the predominant species in North America; manifestations of the infection due to this species range from the mild (young healthy individuals) to severe (immunocompromised patients and the elderly). $B$. divergens is the predominant species in Europe; nearly all symptomatic cases attributed to $B$. divergens occurred among 
asplenic individuals. Definitive diagnosis of babesiosis should be made by microscopic examination of a thin blood smear. PCR is useful in the setting of low parasitemia (e.g., at the onset of symptoms and during convalescence). Serology alone is not diagnostic, but may help determine whether the infection is acute, active, or recent. For patients with mild $B$. microti infection, treatment with atovaquone-azithromycin (Grade 1B) is recommended.

For the babesiosis asymptomatic patients administering antibiotic therapy (Grade 1C) is not recommended. Treatment should be considered if persisted for $\geq 3$ months. For patients with severe $B$. microti infection, initial antimicrobial therapy with clindamycinquinine (Grade 1B) is recommended. The indications for exchange transfusion include high-grade parasitemia $(\geq 10 \%)$, severe anemia (hemoglobin $\leq 10 \mathrm{~g} / \mathrm{dL}$ ), or pulmonary, renal or hepatic compromise. Exchange transfusion should be performed in consultation with experts in hematology and pheresis. $B$. divergens frequently causes fulminant illness and should be considered a medical emergency. Exchange transfusion in consultation with hematology and pheresis expertise (Grade 1C), followed by antimicrobial therapy with clindamycin (and quinine when tolerated) (Grade 1B) is recommended. Administration of antimicrobial therapy for 7 to 10 days is usually sufficient for curative treatment of mild disease. Longer duration of antimicrobial therapy may be needed in cases of persistent and relapsing babesiosis. The immunocompromised patients should be treated for at least six weeks, including two weeks after parasites disappearing from blood smear.

\section{References}

Abdelrahman, RZ, Mohamad, HM, Morsy, ATA, Morsy, TA, 2015: Allergic reactions caused by venom of hymenopterous stinging insects and the role of health care workers. J. Egypt. Soc. Parasitol. 45, 2:403-12.

Abou-Laila, M, Batadoj, D, Salama, A, Munkhjargal, T, Ichikawa-Seki, M, et al, 2014: Ev- aluation of the inhibitory effects of miltefosine on the growth of Babesia and Theileria parasites. Vet Parasitol. 204, 3/4:104-10.

Adham, FK, El-Samie-Abd, EM, Gabre, RM, El Hussein, H, 2010: Detection of tick blood parasites in Egypt using PCR assay II- Borrelia burgdorferi sensu lato. J. Egypt. Soc. Parasitol. 40, 3:553-64.

Alcoba, G, Chappuis, F, 2015: The tropical traveling child: risks and prevention. Rev. Med. Suisse. 11, 473:1023-7

Aufranc S, 3rd, Monahan-Earley, R, et al, 2002: Transfusion medicine illustrated. Morphologic hallmarks of Babesia. Transfusion 42: 1389.

Bajer, A, Alsarraf, M, Bednarska, M, Mohallal, EM, Mierzejewska, EJ, et al, 2014: Babesia behnkei sp. nov., a novel Babesia species infecting isolated populations of Wagner's gerbil, Dipodillus dasyurus, from the Sinai Mountains, Egypt. Parasit. Vectors 7:572-9.

Beattie, JF, Michelson, ML, Holman, PJ, 2002: Acute babesiosis caused by Babesia divergens in a resident of Kentucky. N. Engl. J. Med. 347:697-702.

Birkenheuer, AJ, Whittington, J, Neel, J, Large, E, Barger, A, et al, 2006: Molecular characterization of a Babesia species identified in a North American raccoon. J. Wildl. Dis. 42, 2: 375-80,

Bratton, RL, Corey, R, 2005: Tick-borne disease. Am. Fam. Physician. 71, 12:2323-30.

Chabria, S, Ogbuagu, O. 2015: Fatal multiple deer tick-borne infections in an elderly patient with advanced liver disease. BMJ Case Rep. 2015 Mar 2; 2015. pii: bcr2014208182..

Chiossone, L, Audonnet, S, Chetaille, B, Chasson, L, Farnarier, C, et al, 2012: Protection from inflammatory organ damage in a murine model of hemophagocytic lymphohistiocytosis using treatment with IL-18 binding protein. Front Immunol. 3:239-44.

Clement, ME, Okeke, NL, Hicks, CB, 2015: Fever and rash in a patient with hepatitis. JAMA 314, 4:400-42.

Curno, O, Reader, T, McElligott, AG, Behnake, JM, Barnard, CJ, 2011: Infection before pregnancy affects immunity and response to social challenge in the next generation. Philos. Trans. Roy Soc. Lond. B Biol. Sci. 366, 1583: 3364-74. 
Della-Giustina, D, Laird, TW Jr, Smith, T, 2005: Transfusion-acquired babesiosis in a nonendemic area. Mil. Med. 170:295-9.

Dietrich, G, Dolan, MC, Peralta-Cruz, J, et al, 2006: Repellent activity of fractioned compounds from Chamaecyparis nootkatensis essential oil against nymphal Ixodes scapularis (Acari: Ixodidae). J. Med. Entomol. 43:957-64.

Dobroszycki, J, Herwaldt, BL, Boctor, F, Miller, J, Linden. J, et al, 1999: A cluster of transfusion-associated babesiosis cases traced to a single asymptomatic donor. JAMA 281, 10:92730.

Duh, D, Jelovsek, M, Avsic-Zupanc, T, 2007: Evaluation of an indirect fluorescence immunoassay for the detection of serum antibodies against Babesia divergens in humans. Parasitology 134, Pt 2:179-85.

Eisen, L, Wong, D, Shelus, V, Eisen, RJ, 2013: What is the risk for exposure to vectorborne pathogens in United States national parks? J. Med. Entomol. 50, 2:221-30.

El Bahnasawy, MM, Khalil, HH, Morsy, TA, 2011: Babesiosis in an Egyptian boy acquired from pet dog, and a general review. J. Egypt. Soc. Parasitol. 41, 1:99-108

EI Bahnasawy, MM, Morsy, TA, 2008: Egyptian human babesiosis and general review. J. Egypt. Soc. Parasitol. 38, 1:265-72.

EI Bahy, NM, Bazh, EK, Shaheen, HM, 2015: Efficacy of deltamethrin, diazinon, and ivermectin on Boophilus annulatus ticks (in vitro and in vivo study). Parasitol. Res. 114, 1:29-36.

El Kammah, KM, Oyoun, LM, Abdel-Shafy, S, 2001: Investigation of blood parasites in livestock infested with argasid and ixodid ticks in Egypt. J. Egypt. Soc. Parasitol. 31, 2:365-71

El Kammah, KM, Oyoun, LM, Abdel-Shafy, S, 2007: Detection of microorganisms in the saliva and mid-gut smears of different tick species (Acari: Ixodoidea) in Egypt. J. Egypt. Soc. Parasitol. 37, 2:533-9.

Elsify, A, Sivakumar, T, Nayel, M, Salama, A, Elkhtam, A, et al, 2015: An epidemiological survey of bovine Babesia and Theileria parasites in cattle, buffaloes, and sheep in Egypt. Parasitol. Int. 64, 1:79-85.

Falagas, ME, Klempner, MS, 1996: Babesiosis in patients with AIDS: a chronic infection presenting as fever of unknown origin. Clin. Infect. Dis. 22, 5:809-12.

Falagas, ME, Klempner, MS, 1996: Babesiosis in patients with AIDS: a chronic infection pre- senting as fever of unknown origin. Clin. Infect. Dis. 22:809-14.

Farah, AW, Hegazy, NA, Romany, M, Soliman, YA, Daoud, AM, 2003: Molecular detection of Babesia equi in infected and carrier horses by polymerase chain reaction. Egypt. J. Immunol. 10, 2:73-9.

Fell, E, 2000: An update on Lyme disease and other tick-borne illnesses. Nurse Pract. 25, 10: 38-40, 43-4, 47-8.

Florescu, D, Sordillo, PP, Glyptis, A, et al, 2008: Splenic infarction in human babesiosis: two cases and discussion. Clin. Infect. Dis. 46: e8-12.

Froberg, MK, Dannen, D, Bernier, N, et al, 2008: Case report: spontaneous splenic rupture during acute parasitemia of Babesia microti. Ann. Clin. Lab. Sci. 38:390-8.

Golo, PS, Santos, HA, Perinotto, WM, Quinelato, S, Angelo, IC, et al, 2015: The influence of conidial Pr1 protease on pathogenicity potential of Metarhizium anisopliae senso latu to ticks. Parasitol. Res. 114, 6:2309-15.

Gray, J, Zintl, A, Hildebrandt, A, Hunfeld, K P, Weiss, L, 2010: Zoonotic babesiosis: Overview of the disease and novel aspects of pathogen identity. Ticks Tick Borne Dis. 1, 1:3-10.

Gubernot, DM, Lucey, CT, Lee, KC, et al, 2009: Babesia infection through blood transfusions: reports received by the US Food and Drug Administration, 1997-2007. Clin. Infect. Dis. 48: 25-32.

Guneidy, RA, Shahein, YE, Abouelella, AM, Zaki, ER, Hamed, RR, 2014: Inhibition of the recombinant cattle tick Rhipicephalus (Boophilus) annulatus glutathione S-transferase. Ticks Tick Borne Dis. 5, 5:528-36.

Guswanto, A, Sivakumar, T, Rizk, MA, Elsayed, SA, Youssef, MA, et al, 2014: Evaluation of a fluorescence-based method for antibabesial drug screening. Antimicrob. Agents Chemother. 58, 8:4713-7

Hafez, M, El-Refaii, AH, Michael, SA, 1982: Experimental trans-mission of some blood parasites by Rhipicephalus species in Egypt II- Role of R. turanicus in transmitting Babesia ovis in sheep. J. Egypt. Soc. Parasitol. 12, 2:543-9.

Hatcher, JC, Greenberg, PD, Antique, J, Jimenez-Lucho, VE, 2001: Severe babesiosis in Long Island: review of 34 cases and their complications. Clin. Infect. Dis. 32:1117-24.

Hayes, EB, Piesman, J, 2003: How can we prevent Lyme disease? N. Engl. J. Med. 348:2424. 
Herwaldt, BL, de Bruyn, G, Pieniazek, NJ, et al, 2004: Babesia divergens-like infection, Washington State. Emerg. Infect. Dis. 10:622-9. Hildebrandt, A, Gray, JS, Hunfeld, KP, 2013: Human babesiosis in Europe: what clinicians need to know. Infection 41, 6:1057-72

Hildebrandt, A, Hunfeld KP, 2014: Human babesiosis: A rare but potentially dangerous zoonosis. Dtsch. Med. Wochenschr. 139, 18:957-62. Homer, MJ, Aguilar-Delfin, I, Telford, SR, 3 rd, et al, 2000: Babesiosis. Clin. Microbiol. Rev. 13:451-9.

Hunfeld, KP, Hildebrandt, A, Gray, JS, 2008: Babesiosis: Recent insights into an ancient disease. Int. J. Parasitol. 38, 11:1219-37.

Katz, TM, Miller, JH, Hebert, AA, 2008: Insect repellents: Historical perspectives and new developments. J. Am. Acad. Dermatol. 58:86574.

Kjemtrup, AM, Lee, B, Fritz, CL, et al, 2002: Investigation of transfusion transmission of a WA1-type babesial parasite to a premature infant in California. Transfusion 42:1482-9.

Krause, PJ, Gewurz, BE, Hill, D, et al, 2008: Persistent and relapsing babesiosis in immunocompromised patients. Clin. Infect. Dis. 46:3708.

Krause, PJ, Lepore, T, Sikand, VK, et al, 2000: Atovaquone and azithromycin for the treatment of babesiosis. N. Engl. J. Med. 343:14549.

Krause, PJ, Lepore, T, Sikand, VK, Gadbaw, J Jr, Burke, G, et al, 2000: Atovaquone and azithromycin for the treatment of babesiosis. N. Engl. J. Med. 343, 20:1454-8.

Kuwayama, DP, Briones, R, 2008: Spontaneous splenic rupture caused by Babesia microti infection. Clin. Infect. Dis. 46:e92-8.

Lappin, MR, Chandrashekar, R, Stillman, B, Liu, J, Mather, TN, 2015: Evidence of Anaplasma phagocytophilum and Borrelia burgdorferi infection in cats after exposure to wildcaught adult Ixodes scapularis. J. Vet. Diagn. Invest. 2015 Jul 15. pii: 1040638715593598.

Leiby, DA, 2006: Babesiosis and blood transfusion: flying under the radar. Vox. Sang. 90:15762.

Leschnik, M, Feiler, A, Duscher, GG, Joachim, A, 2013: Effect of owner-controlled acaricidal treatment on tick infestation and immune response to tick-borne pathogens in naturally infested dogs from Eastern Austria. Parasit. Vectors 6:62-76.
Mahmmod, Y, 2013: Natural Babesia bovis infection in water buffaloes (Bubalus bubalis) and crossbred cattle under field conditions in Egypt: A preliminary study. J. Arthropod Borne Dis. 8, 1: 1-9.

Mahmoud, MS, Kandil, OM, Nasr, SM, Hendawy, S, Habeeb, S, et al, 2015: Serological \& molecular diagnostic surveys combined with examining hematological profiles suggests increased levels of infection and hematological response of cattle to babesiosis infections compared to native buffaloes in Egypt. Parasit. Vectors Jun 12;8:319.doi:10.1186/s13071-015-0928-9.

Marathe, A, Tripathi, J, Handa, V, Date, V, 2005: Human babesiosis, a case report. Indian $J$. Med. Microbiol. 23, 4:267-9.

Maronpot, RR, Guindy, E, 1970: Preliminary study of Babesia gibsoni Patton in wild carnivores and domesticated dogs in Egypt. Am. J. Vet. Res. 31, 4:797-9.

Mazyad, SA, Khalaf, SA, 2002: Studies on Theileria and Babesia infecting live and slaughtered animals in Al Arish and El Hasanah, North Sinai Governorate, Egypt. J. Egypt. Soc. Parasitol. 32, 2: 601-10.

Mazyad, SA, Shoukry, NM, El-Alfy, NM, 2010: Efficacy of Ixodes ricinus as a vector of zoonotic babesiosis in Sinai Peninsula, Egypt. J. Egypt. Soc. Parasitol. 40, 2:499-514.

Michael, SA, El Refaii, AH, 1982: The effect of imidocarb dipropionate on Babesia ovis infection in sheep. Trop. Anim. Hlth. Prod. 14, 1:1-2. Michael, SA, Morsy, TA, Montasser, MF, 1987: A case of human babesiosis (Preliminary case re-port in Egypt). J. Egypt. Soc. Parasitol. 17, 1:409-10.

Morsy, TA, Haridy, FM, 2000: Effect of ivermectin on the brown dog tick, Rhipicephalus sanguineus. J. Egypt. Soc. Parasitol. 30, 1:11724.

Na, YJ, Chai, JY, Jung, BK, Lee, HJ, Song, J Y, et al, 2014: An imported case of severe falciparum malaria with prolonged hemolytic anemia clinically mimicking a co-infection with babesiosis. Korean J. Parasitol. 52, 6:667-72.

Nagaty, HF, 1947: Some new and rare records of piroplasmosis with a list of the species of Babesia and Theileria so far recorded from Egypt. Vet. Rec. 59, 11:145-7.

Nayel, M, El-Dakhly, KM, Aboulaila, M, Elsify, A, Hassan, H, et al, 2012: The use of different diagnostic tools for Babesia and Theileria 
parasites in cattle in Menofia, Egypt. Parasitol. Res. 111, 3: 1019-24

Panduranga, V, Kumar, A, 2014: Severe babesiosis presenting as acute respiratory distress syndrome in an immunocompetent patient. Conn. Med. 78, 5:289-91

Panella, NA, Dolan, MC, Karchesy, JJ, et al, 2005: Use of novel compounds for pest control: insecticidal and acaricidal activity of essential oil components from heartwood of Alaska yellow cedar. J. Med. Entomol. 42:352-9.

Park, H, Hong, SH, Kim, K, Cho, SH, Lee, W J, et al, 2015: Characterizations of individual mouse red blood cells parasitized by Babesia microti using 3-D holographic microscopy. Sci. Rep. 2015 Jun 3; 5:10827. doi:10.1038/ srep 10827.

Persing, DH, Herwaldt, BL, Glaser, C, et al, 1995: Infection with a Babesia-like organism in north-ern California. N. Engl. J. Med. 332:298304.

Persing, DH, Mathiesen, D, Marshall, WF, et $\boldsymbol{a l}$, 1992: Detection of Babesia microti by polymerase chain reaction. J. Clin. Microbiol. 30: 2097-105.

Qazzafi, Z, Thiruchunapalli, D, Birkenhead, D, Bell, D, Sandoe, JA, 2007: Invasive Cryptococcus neoformans infection in an asplenic patient. J. Infect. 55, 6:566-8.

Rosenblatt, JE, 2009: Laboratory diagnosis of infections due to blood and tissue parasites. Clin. Infe- ct. Dis. 49, 7:1103-8.

Saleh, MA, 2009: Erythrocytic oxidative damage in crossbred cattle naturally infected with Babesia bigemina. Res. Vet. Sci. 86, 1:43-8.

Sethi, Sonia, David, A, Hemant Kesarwala, Tolan, RW, Jr, 2009: Probable Congenital Babesiosis in Infant, New Jersey, USA. Emerg. Infect. Dis. 15, 5:788-91.

Shaio, MF, Yang, KD, 1997: Response of babesiosis to a combined regimen of quinine and azithrom-ycin. Trans. R. Soc. Trop. Med. Hyg. 91:214-8.

Shapiro. R, 2012: Prevention of vector transmitted diseases with clove oil insect repellent. J. Pediatr. Nurs. 27, 4:346-9.

Shih, CM, Wang, CC, 1998: Ability of azithromycin in combination with quinine for the elimination of babesial infection in humans. Am. J. Trop. Med. Hyg. 59, 4:509-12.

Stafford, KC, 2009: Tick Management Handbook. http://www.cdc.gov/ncidod/dvbid/lyme/resources/handbook.pdf (Accessed July 14, 2009).
Stowell, CP, Gelfand, JA, Shepard, JA, Kratz, A, 2007: Case records of the Massachusetts General Hospital. Case 17-2007: A 25-year-old woman with relapsing fevers and recent onset of dyspnea. N. Engl. J. Med. 356:2313-8.

Vannier, E, Gewurz, BE, Krause, PJ, 2008: Human babesiosis. Infect. Dis. Clin. North Am. 22:469-74.

Vannier, E, Krause, PJ, 2009: Update on babesiosis. Interdiscip. Perspect. Infect. Dis. 984568-76

Vazquez, M, Muehlenbein, C, Cartter, M, et al, 2008: Effectiveness of personal protective measures to prevent Lyme disease. Emerg. Infect. Dis. 14:210-9.

Vercammen, F, De Deken, R, Maes, L, 1995: Clinical and serological observations on experimental infections with Babesia canis and its diagnosis using the IFAT. Parasite (Paris, France) 2, 4:407-10.

Vial, HJ, Gorenflot, A, 2006: Chemotherapy against babesiosis. Vet. Parasitol. 138, 1/2:14760 .

Weiss, LM, Wittner, M, Tanowitz, HB, 2001: The treatment of babesiosis. N. Engl. J. Med. 344:773-9.

White, NJ, 2007: Cardiotoxicity of antimalarial drugs. Lancet Infect. Dis. 7:549-52.

Wilson, M, Glaser, KC, Adams-Fish, D, Boley, M, Mayda, M, et al, 2015: Development of droplet digital PCR for the detection of Babesia microti and Babesia duncani. Exp. Parasitol. 149:24-31.

Wormser, GP, Dattwyler, RJ, Shapiro, ED, et al, 2006: The clinical assessment, treatment, and prevention of Lyme disease, human granulocytic anaplasmosis, and babesiosis: clinical practice guidelines by the Infectious Diseases Society of America. Clin. Infect. Dis. 43:1089-98.

Wormser, GP, Prasad, A, Neuhaus, E, Joshi, S, Nowakowski, J, et al, 2010: Emergence of resistance to azithromycin-atovaquone in immuno-compromised patients with Babesia microti infection. Clin. Infect. Dis. 50, 3:381-6.

Youssef, AI, Uga, S. 2014: Review of parasitic zoonoses in Egypt. Trop. Med. Hlth. 42, 1:3-14.

Zintl, A, Mulcahy, G, Skerrett, H, et al, 2003: Babesia divergens, a bovine blood parasite of veterinary and zoonotic importance. Clin. Microbiol. Rev.16:622-30. 